\title{
Abirateron in combinatie met androgeendeprivatietherapie bij patiënten met hormoonnaïef gemetastaseerd prostaatcarcinoom
}

\author{
Is het tijdperk van androgeendeprivatie-monotherapie voorbij?
}

\author{
Robert J. van Soest ${ }^{1}$ • Pim J. van Leeuwen ${ }^{1}$ • Joost L. Boormans ${ }^{1}$. Chris H. Bangma ${ }^{1}$ \\ Published online: 15 January 2018 \\ (c) The Author(s) 2018
}

\section{Samenvatting}

Data van twee gerandomiseerde studies hebben aangetoond dat abirateron plus prednison, gecombineerd met androgeendeprivatietherapie (ADT) de overleving verbetert van patiënten met primair gemetastaseerd hormoonnaïef prostaatcarcinoom (mHSPC) in vergelijking met ADT alleen (hazardratio 0,62 en 0,63). De belangrijkste bijwerkingen (hypokaliëmie en hypertensie) waren mild (graad 1-2) en goed te behandelen. Voor patiënten met hoogrisico lokaal gevorderd of lymfogeen gemetastaseerd prostaatcarcinoom zonder metastasen op afstand was er een winst in progressievrije overleving, maar zijn de data nog te immatuur om overlevingswinst aan te tonen. Voor patiënten met mHSPC zou abirateron plus prednison en ADT als een nieuwe standaardbehandeling beschouwd kunnen worden.

Trefwoorden abirateron · androgeendeprivatietherapie $\cdot$ gemetastaseerd hormoonnaïef prostaatcarcinoom

\section{Abiraterone and androgen-deprivation therapy for hormone-sensitive metastatic prostate cancer} Is the era of androgen deprivation monotherapy over?

\begin{abstract}
Two randomized trials have shown that abiraterone plus prednisone and androgen-deprivation therapy (ADT) improved survival as compared with ADT alone in patients with de novo metastatic hormone-sensitive prostate cancer (mHSPC) (hazard ratio 0.62-0.63). The most important side-effects (hypokalemia and hypertension) were mild (mostly grade 1-2) and easy to manage. For patients with high-risk locally advanced disease or lymph node metastases abiraterone plus prednisone and ADT increased progression-free survival. However, the data is still immature to show overall survival benefit in this patient subgroup. For patients with mHSPC, combination therapy with abiraterone plus prednisone and ADT can be considered a new standard of care.
\end{abstract}

Keywords abiraterone $\cdot$ androgen-deprivation therapy $\cdot$ metastatic hormone-sensitive prostate cancer

dr. Robert J. van Soest

r.vansoest@erasmusmc.nl

1 afdeling Urologie, Erasmus Medisch Centrum en Kanker Instituut, Rotterdam, Nederland 
Figuur 1 Steroïdsynthese uit cholesterol en rol-CYP17A1 (substraat abirateron)

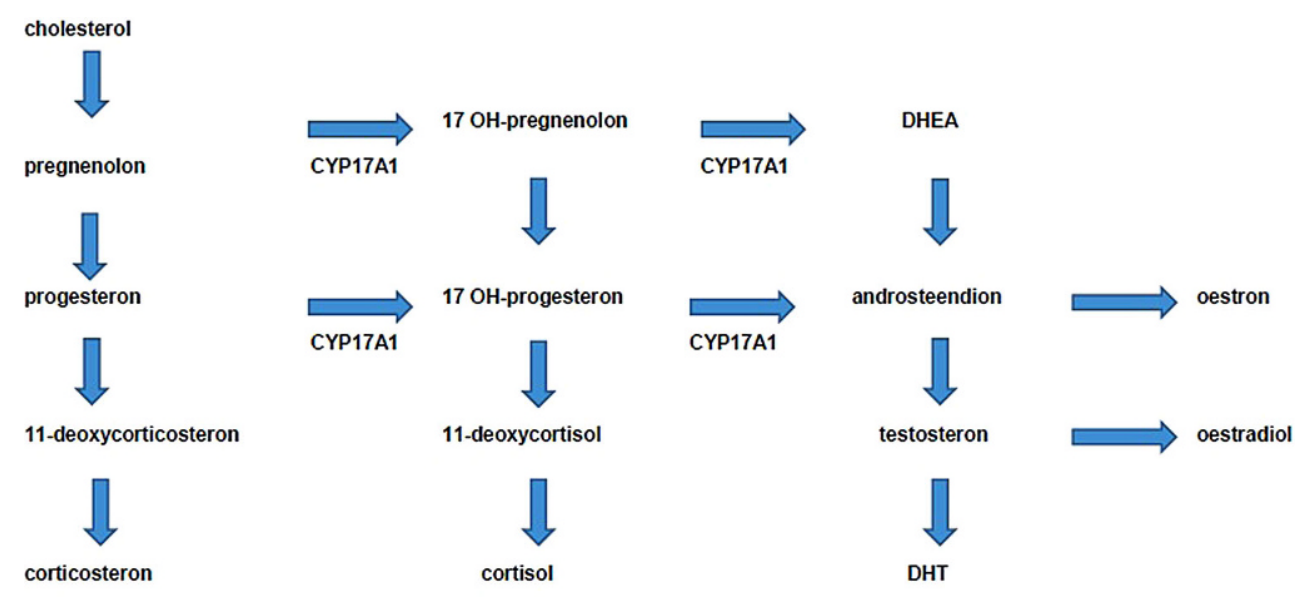

\section{Introductie}

Begin juni 2017 zijn op de jaarlijkse bijeenkomst van de American Society of Clinical Oncology (ASCO) de resultaten gepresenteerd van de LATITUDE- en STAMPEDEstudies. Beide studies onderzochten de toevoeging van abirateron aan standaard androgeen deprivatietherapie (ADT) bij mannen met hormoonnaïef gemetastaseerd prostaatkanker (mHSPC). Deze twee gerandomiseerde studies, gepubliceerd in de New Engeland Journal of Medicine, lieten zien dat het toevoegen van abirateron plus prednison aan standaard-ADT leidde tot een significante overlevingswinst in vergelijking met ADT alleen bij mannen met mHSPC.

Sinds enkele jaren is abirateron gecombineerd met prednison een van de geregistreerde behandelopties voor patiënten met gemetastaseerd castratieresistent prostaatcarcinoom (mCRPC). De COU-AA-302- en COU-AA-301-studies toonden een overlevingswinst aan van respectievelijk 4,4 en 4,6 maanden bij patiënten voor en na behandeling met docetaxel chemotherapie $[1,2]$. De winst in totale en in progressievrije overleving (PFS) waren indicatief voor het feit dat het blokkeren van androgeensynthese door het enzym CYP17A1 (fig. 1), het moleculair substraat van abirateron, zinvol is bij patiënten die reeds ADT krijgen. Androgeensynthese ondanks optimale ADT kan optreden in zowel de bijnier - uit steroïdprecursors - als in de tumorcellen zelf, die op deze manier een resistentiemechanisme ontwikkelen [3]. Het succes van abirateron bij mCRPC-patiënten heeft geleid tot het testen van dit middel bij patiënten met hormoonnaïef gemetastaseerd en hoogrisico lokaal gevorderd prostaatcarcinoom.

Dit artikel heeft als doel inzicht te geven in de effectiviteit en mogelijke bijwerkingen van abiraterone plus prednison in combinatie met standaard ADT, bij de behandeling van mHSPC en hoogrisico lokaal gevorderd prostaatcarcinoom.

\section{Abirateron bij hormoonnaïef lokaal gevorderd en gemetastaseerd prostaatcarcinoom}

De LATITUDE-studie is een gerandomiseerde multicenterstudie, waarin de combinatie van ADT met abirateron $(1 \mathrm{dd} 1.000 \mathrm{mg})$ plus prednison $(1 \mathrm{dd} 5 \mathrm{mg})$ versus ADT met placebo is onderzocht bij 1.190 patiënten met een primair mHSPC. Patiënten werden geïncludeerd wanneer twee van de drie volgende risicofactoren aanwezig waren: een Gleason-score $\geq 8$, drie of meer botmetastasen of de aanwezigheid van viscerale metastasen. Patiënten werden vervolgens gerandomiseerd volgens een 1:1-ratio naar ADT met abirateron $(1.000 \mathrm{mg})$ plus prednison $(5 \mathrm{mg})(n=597)$ versus ADT met placebo $(n=602)$. Primaire eindpunten van de studie waren overleving en radiologische progressievrije overleving (rPFS). Secundaire eindpunten waren: progressie van pijnklachten, biochemische progressie, symptomatisch skeletal-related event (SRE), tijd tot start van de chemotherapie en aanvullende prostaatkankertherapie. Na een mediane follow-up van 30,4 maanden hadden patiënten behandeld met ADT plus abirateron en prednison een $38 \%$ lager risico op sterfte dan patiënten behandeld met ADT plus placebo (hazard ratio $[\mathrm{HR}]=0,62 ; 95 \%$ betrouwbaarheidsinterval $[\mathrm{BI}]: 0,51-0,76)$. De mediane overleving in de combinatiegroep was niet bereikt in vergelijking met 34,7 maanden in de ADT plus placebo arm. Er werd een $53 \%$ reductie in rPFS of overlijden aangetoond in de groep behandeld met ADT plus abirateron vergeleken met ADT plus placebo. Ook alle secundaire eindpunten van de studie waren positief. 
Een belangrijke limitatie van de studie is dat slechts $11 \%$ van de patiënten in de ADT plus placebo arm werden behandeld met abirateron als eerstelijns therapie bij castratieresistente ziekte, en dat $21 \%$ van de patiënten zelfs helemaal geen vervolgtherapie ontving. De controlearm van de studie is dus niet te vergelijken met de huidige standard of care waar abirateron/enzalutamide standaard eerstelijns therapie is. In plaats van een vergelijking tussen vroege versus late behandeling met abirateron, is in LATITUDE vroege behandeling met abirateron vergeleken met 'geen behandeling'. Hierbij is interessant dat de overlevingswinst het meest uitgesproken lijkt te zijn voor de patiënten geïncludeerd in Oost-Europa, hetgeen te maken zou kunnen hebben met een mindere beschikbaarheid van vervolg therapieën in vergelijking met West-Europa.

De STAMPEDE-studie is een gerandomiseerde multicenter trial bij 1.917 patiënten. In deze trial is het effect van de combinatie van ADT met abirateron plus prednison vergeleken met het effect van ADT alleen. Het grote verschil met de LATITUDE-studie is dat in de STAMPEDEstudie ook niet-gemetastaseerde patiënten zijn geïncludeerd (high-risk locally advanced NOMO (27\%) en N1MO (20\%)). Patiënten met lokaal gevorderd prostaatcarcinoom werden geïncludeerd wanneer twee van de volgende kenmerken aanwezig waren: klinisch stadium T3 $/ 4, \mathrm{PSA} \geq 40 \mathrm{ng} / \mathrm{ml}$, of Gleason-score 8-10. Lokale uitwendige radiotherapie was opgenomen in het studieprotocol: deze behandeling was verplicht voor de N0M0-patiënten en optioneel voor de N1M0-patiënten. Patiënten met een status na eerdere therapie (ca $5 \%$, radicale prostatectomie of radiotherapie) konden deelnemen indien meer dan een van de volgende kenmerken aanwezig waren: PSA $\geq 4 \mathrm{ng} / \mathrm{ml}$ en een PSA-verdubbelingstijd (PSDT) $<6$ maanden, PSA $\geq 20 \mathrm{ng} / \mathrm{ml}$, N1-, of M1-ziekte. Patiënten werden 1:1 gerandomiseerd naar ADT $\geq 2$ jaar $(n=957)$ en ADT plus abirateron en prednison. Het primaire eindpunt was overleving. Het intermediate primaire eindpunt was failure-free survival (FFS), waarbij failure werd gedefinieerd als: biochemische progressie, lokale progressie, het ontwikkelen van metastasen of dood door prostaatkanker. Secundaire eindpunten waren toxiciteit en SRE's. Behandeling met abirateron plus prednison met ADT resulteerde in een relatieve overlevingswinst van $37 \%$ in vergelijking met ADT alleen (HR 0,63; $95 \%-\mathrm{BI}=0,52-0,76 ; p<0,001)$.

Patiënten die waren behandeld in de combinatiearm hadden een significant betere FFS, met een HR van 0,29 (95\%$\mathrm{BI}=0,25-0,34 ; p<0,001)$. Ook hier waren alle secundaire eindpunten positief. Een kanttekening is dat de totale overleving voor de subgroep met M0-patiënten nog immatuur is door een te laag aantal events. Wel was er een trend in het voordeel van abirateron met ADT voor FFS.

\section{Bijwerkingen van abirateron}

Abirateron inhibeert het enzym CYP17A1 en daarmee de productie van testosteron (fig. 1). Gebruik van abirateron om de testosteronproductie te reduceren, heeft verschillende ongewenste fysiologische veranderingen tot gevolg, waaronder een verlaging van cortisol en compensatoire stijging van adrenocorticotroop hormoon (ACTH) [4]. Deze ACTHstijging resulteert in verschuivingen in de mineralocorticoïden, met als direct gevolg klinische bijwerkingen, zoals hypokaliëmie, hypertensie en oedeem [5]. Om deze bijwerkingen als direct gevolg van de CYP17A1-blokkade tegen te gaan, dient abirateron gecombineerd te worden met een glucocorticoïd, zoals prednison. In studies met mCRPC-patiënten leidde het gebruik van abirateron tot een verlaging van het serumcortisol tot onder de normaalwaarde en steeg de mediane ACTH-waarde van $17 \mathrm{pg} / \mathrm{mL}$ naar $124 \mathrm{pg} / \mathrm{ml}$ [4]. Indien een lage dosis prednison wordt toegevoegd aan abirateron wordt de door abirateron geïndiceerde verandering in het cortisol- en ACTH-gehalte genormaliseerd [1].

Ook glucocorticoïden kunnen echter verschillende bijwerkingen veroorzaken indien ze langdurig in hoge doses worden gebruikt, bijvoorbeeld bij de behandeling van verschillende auto-immuunziekten, zoals reumatoïde artritis, en de ziekte van Crohn. Bekende aan glucocorticoïd gerelateerde bijwerkingen zijn: een verstoord botmetabolisme, onderdrukking van de immuunrespons, spierzwakte, een verhoogd risico op hyperglykemie en diabetes mellitus. In een groep van 2.267 patiënten met mCRPC die behandeld werden met een lage dosis prednison plus abirateron, toonden Fizazi et al. aan dat de incidentie van glucocorticoïd-gerelateerde bijwerkingen echter zeer laag was na een follow-up van meer dan 30 maanden [6].

De toxiciteit en veiligheid van abirateron is uitgebreid geëvalueerd in de eerdere COU-AA-302- en COU-AA301-studies bij patiënten met mCRPC, en recent in de LATITUDE- en STAMPEDE-studies. In zowel de COUAA-302- als de COU-AA-301-studie was de incidentie van mineralocorticoïd-gerelateerde bijwerkingen hoger in de groep patiënten die was behandeld met abirateron plus prednison dan in de groep die alleen met prednison was behandeld (tabel 1).

Het is belangrijk om te vermelden dat de meeste aan mineralocorticoïden gerelateerde bijwerkingen graad 1 en 2 waren (13\% graad 1-2 hypokaliëmie en $8 \%$ hypertensie in COU-AA-301). De incidentie van graad 3-4 mineralocorticoïd-gerelateerde bijwerkingen was laag en deze waren goed te behandelen (hypertensie 1\%, hypokaliëmie $3 \%$ en oedeem $2 \%$ ). Uit follow-upstudies kwamen na meer dan 24 maanden therapie geen andere bijwerkingen naar voren. Ook in de LATITUDE-studie waren de meest gerapporteerde mineralocorticoïd-gerelateerde bijwerkingen hypertensie en hypokaliëmie (graad 3-4: $20 \%$ en 10\% res- 
Tabel 1 Bijwerkingen van abirateron plus prednison in de COU-AA-302- en COU-AA-301-studies

\begin{tabular}{|c|c|c|c|c|}
\hline \multirow[b]{2}{*}{ bijwerking $n(\%)$} & \multicolumn{2}{|c|}{ COU-AA-302 [10] } & \multicolumn{2}{|c|}{ COU-AA-301 [1] } \\
\hline & $\begin{array}{l}\text { AA + prednison } \\
(n=542)\end{array}$ & $\begin{array}{l}\text { prednison } \\
(n=540)\end{array}$ & $\begin{array}{l}\mathrm{AA}+\text { prednison } \\
(n=791)\end{array}$ & $\begin{array}{l}\text { prednison } \\
(n=394)\end{array}$ \\
\hline \multicolumn{5}{|l|}{ graad 1-4 } \\
\hline oedeem & $150(28)$ & $127(24)$ & $241(31)$ & $88(22)$ \\
\hline hypokaliëmie & $91(17)$ & $68(13)$ & $135(17)$ & $33(8)$ \\
\hline hypertensie & $118(22)$ & $71(13)$ & 77 (10) & $31(8)$ \\
\hline \multicolumn{5}{|l|}{ graad 3 en 4} \\
\hline oedeem & $4(<1)$ & $9(2)$ & $18(2)$ & $4(1)$ \\
\hline hypokaliëmie & $13(2)$ & $10(2)$ & $30(3)$ & $3(1)$ \\
\hline hypertensie & $21(4)$ & $16(3)$ & $10(1)$ & $1(<1)$ \\
\hline
\end{tabular}

$A A$ abirateronacetaat

Tabel 2 Bijwerkingen van abirateron plus prednison gecombineerd met ADT in de LATITUDE- en STAMPEDEstudies

\begin{tabular}{lcl}
\hline bijwerking $n(\%)$ & LATITUDE graad $\geq 3$ & STAMPEDE graad $\geq 3$ \\
\hline hypertensie & $121(20)$ & $44(5)$ \\
hypokaliëmie & $62(10)$ & $12(1)$ \\
ALAT stijging & $33(5)$ & $53(6)$ \\
ASAT stijging & $26(4)$ & $10(1)$ \\
hyperglykemie & $27(4)$ & niet bekend \\
\hline
\end{tabular}

pectievelijk) (tabel 2). Dit is aanzienlijk hoger dan in de COU-AA-301- en -302-studies, hetgeen meest waarschijnlijk wordt veroorzaakt door een strikter classificatiesysteem voor het rapporteren van bijwerkingen (NCI-CTCAE versie 4 in plaats van 3$)$. Slechts twee patiënten moesten de behandeling staken vanwege hypokaliëmie en mortaliteit door hypokaliëmie trad niet op. De incidentie van aan mineralocorticoïd gerelateerde bijwerkingen was in de STAMPEDEstudie een stuk lager ( $5 \%$ hypertensie en $1 \%$ hypokaliëmie) (tab. 2). Deze bijwerkingen kwamen ook voor in de groep die alleen ADT kreeg en waren goed te behandelen.

\section{Monitoring en interacties}

Bij de behandeling met abirateron plus prednison en ADT dienen potentiële bijwerkingen gemonitord te worden, zodat deze tijdig opgespoord en behandeld kunnen worden. Er dient maandelijks een bloeddruk- en kaliumcontrole plaats te vinden. De leverfuncties worden in de eerste drie maanden iedere twee weken gecontroleerd en daarna maandelijks. Hypokaliëmie kan eenvoudig behandeld worden door middel van kaliumsuppletie en hypertensie kan behandeld worden met een antihypertensivum. Bij het optreden van leverfunctiestoornissen (ALAT/ASAT $\geq 5 \times$ de normaalwaarde of bilirubine $\geq 3 \times$ de normaalwaarde) dient de behandeling gestaakt te worden. Wanneer de leverwaarden weer dalen $(<2,5 \times$ de normaalwaarde voor ALAT/ ASAT of $<1,5 \times$ de normaalwaarde voor bilirubine) dient de behandeling hervat te worden met een gereduceerde dosis van $750 \mathrm{mg}$. Indien er wederom levertoxiciteit optreedt dient de dosis verder gereduceerd te worden tot $500 \mathrm{mg}$.

Abirateron is gecontraindiceerd bij patiënten met ernstige leverfunctiestoornissen (Child-Pughscore 10-15). Ook is voorzichtigheid geboden bij patiënten met een linkerventrikelejectiefractie (LVEF) van $<50 \%$ of hartfalen NYHA-klasse II-IV, omdat de veiligheid van abirateron bij deze patiënten niet is vastgesteld. Behandeling van deze patiënten met abirateron is daarom niet raadzaam. Verder dient de combinatie met sterke CYP3A4-inductoren (zoals carbamazepine, fenytö̈ne, fenobarbital, rifampicine, sintjanskruid) vermeden te worden, omdat deze de plasmaconcentratie van abirateron kunnen verlagen. Tab. 3 geeft een samenvatting van de indicatie, behandeling en monitoring van patiënten met mHSPC die in aanmerking komen voor behandeling met abirateron plus prednison en ADT.

\section{Therapeutische implicaties voor de uroloog}

Op basis van de LATITUDE- en STAMPEDE-studies zou abirateron plus een lage dosis prednison in combinatie met ADT de nieuwe standaard kunnen worden voor het primair mHSPC. Voor M0-patiënten en eerder lokaal behandelde patiënten zijn de data op dit moment nog te immatuur om therapeutische consequenties aan te verbinden. De M1-patiënten behandeld met abirateron en ADT is dezelfde groep patiënten waarbij recent ADT plus zes kuren docetaxel de nieuwe standaard behandeling werd naar aanleiding van de CHAARTED-data en de eerder gepubliceerde data van de STAMPEDE-studie [7-9]. De overlevingswinst met zes ku- 
Tabel 3 Indicatie, behandeling en follow-up: ADT plus abirateron plus prednison

\begin{tabular}{lll}
\hline indicatie & behandeling & follow-up \\
\hline primair mHSPC & start ADT + abiraterone 1.000 mg + prednison 5 mg & - PSA in week 6, 12, 18, 24, hierna iedere 12 weken \\
$\begin{array}{l}\text { Gleason-score } \geq 8 \\
\geq 3 \text { botmetastasen }\end{array}$ & & - imaging (CT/bot-scan): bij symptomen/progressie \\
$\begin{array}{l}\text { viscerale metastasen } \\
(2 \text { van de 3) }\end{array}$ & - bloeddruk- en kaliumcontrole: maandelijks \\
& alternatief: ADT + 6 kuren docetaxel & - leverenzymen: iedere 2 weken in de eerste 3 maanden, \\
&
\end{tabular}

ren docetaxel plus ADT is ongeveer vergelijkbaar (24-27\% relatieve risicoreductie op overlijden) met ADT in combinatie met abirateron plus lage dosis prednison. Gezien het ontbreken van vergelijkende studies tussen abirateron en docetaxel, en het gunstigere bijwerkingenprofiel van abiraterone in combinatie met de orale toediening, ligt het voor de hand dat abirateron voor veel patiënten de te prefereren behandelingsoptie is. Echter is de behandelduur langer ( 2 jaar voor abiraterone versus 18 weken voor docetaxel) en zijn de kosten aanzienlijk hoger. Door de langere duur van de behandeling met abirateron zullen patiënten voor langere periode blootgesteld worden aan de bijwerkingen en intensieve controles met een mogelijke impact op de kwaliteit van leven. Echter heeft een recent gepresenteerd abstract (ESMO 2017) over de kwaliteit van leven data van de LATITUDE-studie laten zien dat abirateron plus prednison en ADT de achteruitgang in kwaliteit van leven (gemeten middels de FACT-P- score) remt in vergelijking met ADT en placebo $(\mathrm{HR}=0,85 ; 95 \%-\mathrm{BI}=0,74-0,99 ; p=0,03)$. Ook verminderde abirateron pijnscores in vergelijking met placebo [10].

De effectiviteit van vervolgbehandelingen na abirateron voor mHSPC is nog onduidelijk. Het ligt echter voor de hand dat bij patiënten die castratie-resistent worden na behandeling met abirateron plus ADT, chemotherapie de te prefereren behandelingsoptie is en geen androgeenreceptor gericht middel zoals abirateron of enzalutamide.

Met de introductie van abirateron in combinatie met ADT voor de behandeling van primair mHSPC zal de therapie weer als poliklinische behandeling worden aangeboden. Door de jarenlange ervaring die veel urologen hebben met het geven van standaard-ADT en het gunstige bijwerkingenprofiel van abirateron, is abirateron een middel dat potentieel gegeven kan worden door de uroloog. Vanzelfsprekend dient de indicatie voor start van abirateron plus ADT gesteld te worden in een multidisciplinaire setting, waarin zowel urologen als internist-oncologen participeren, hetgeen momenteel al gebruikelijk is bij de behandeling van het gemetastaseerd prostaatcarcinoom. Indien een internistoncoloog of een uroloog met specifieke interesse voor systeemtherapie zich verdiept in het werkingsmechanisme en de potentiële toxiciteit van abirateron, kunnen deze patiënten langere tijd behandeld worden met als doel het moment tot castratieresistente ziekte uit te stellen en de overleving te verbeteren.

\section{Conclusie}

Abirateron plus prednison gecombineerd met ADT verbetert de overleving van patiënten met primair mHSPC, met een relatief gunstig bijwerkingenprofiel.

Open Access This article is distributed under the terms of the Creative Commons Attribution 4.0 International License (http:// creativecommons.org/licenses/by/4.0/), which permits unrestricted use, distribution, and reproduction in any medium, provided you give appropriate credit to the original author(s) and the source, provide a link to the Creative Commons license, and indicate if changes were made.

\section{Literatuur}

1. Bono JS de, Logothetis CJ, Molina A, et al. Abiraterone and increased survival in metastatic prostate cancer. $\mathrm{N}$ Engl $\mathrm{J}$ Med. 2011;364:1995-2005.

2. Ryan CJ, Smith MR, Fizazi K, et al. Abiraterone acetate plus prednisone versus placebo plus prednisone in chemotherapy-naive men with metastatic castration-resistant prostate cancer (COU-AA-302): final overall survival analysis of a randomised, double-blind, placebo-controlled phase 3 study. Lancet Oncol. 2015;16:152-60.

3. Soest RJ van, Royen ME van, Morree ES de, et al. Cross-resistance between taxanes and new hormonal agents abiraterone and enzalutamide may affect drug sequence choices in metastatic castrationresistant prostate cancer. Eur J Cancer. 2013;49:3821-30.

4. Attard G, Reid AH, Auchus RJ, et al. Clinical and biochemical consequences of CYP17A1 inhibition with abiraterone given with and without exogenous glucocorticoids in castrate men with advanced prostate cancer. J Clin Endocrinol Metab. 2012;97:507-16.

5. Attard G, Reid AH, Yap TA, et al. Phase I clinical trial of a selective inhibitor of CYP17, abiraterone acetate, confirms that castrationresistant prostate cancer commonly remains hormone driven. J Clin Oncol. 2008;26:4563-71.

6. Fizazi K, Chi KN, Bono JS de, et al. Low incidence of corticosteroid-associated adverse events on long-term exposure to low-dose prednisone given with abiraterone acetate to patients with metastatic castration-resistant prostate cancer. Eur Urol. 2016;70:438-44.

7. James ND, Sydes MR, Clarke NW, et al. Addition of docetaxel, zoledronic acid, or both to first-line long-term hormone therapy in prostate cancer (STAMPEDE): survival results from an adaptive, multiarm, multistage, platform randomised controlled trial. Lancet. 2016:387:1163-77. 
8. Sweeney CJ, Chen YH, Carducci M, et al. Chemohormonal therapy in metastatic hormone-sensitive prostate cancer. N Engl J Med. 2015;373:737-46.

9. Soest RJ van, Wit R de. Irrefutable evidence for the use of docetaxel in newly diagnosed metastatic prostate cancer: results from the STAMPEDE and CHAARTED trials. Bmc Med. 2015;13:304.

10. Chi K, Protheroe A, Rodriguez AA, et al. Benefits of abiraterone acetate plus prednisone $(\mathrm{AA}+\mathrm{P})$ when added to androgen deprivation therapy $(\mathrm{ADT})$ in LATITUDE on patient $(\mathrm{Pt})$ reported outcomes (PRO). Ann Oncol. 2017;28(Supp 5):269. dr. Robert J. van Soest aios urologie

dr. Pim J. van Leeuwen aios urologie

dr. Joost L. Boormans uroloog

prof. dr. Chris H. Bangma uroloog 
Hier staat een advertentie.

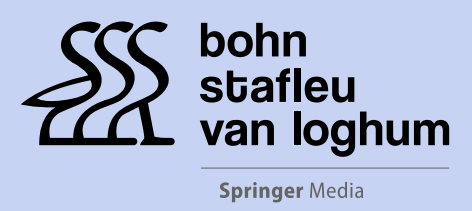

Houten 2018 